\title{
LIE ALGEBRAS ASSOCIATED WITH A STANDARD QUADRUPLET AND PREHOMOGENEOUS VECTOR SPACES
}

\author{
By \\ Nagatoshi SASANO
}

\begin{abstract}
By using the theory of Lie algebras associated with a standard quadruplet, we can embed an arbitrary reductive prehomogeneous vector space with completely reducible representation into some graded Lie algebra. The purpose of this paper is to study properties of graded Lie algebras which correspond to the prehomogeneity condition of triplets. Moreover, we give another proof of castling transformation as an application.
\end{abstract}

\section{Introduction}

Let $(G, \rho, V)$ be a triplet which consists of a connected algebraic group $G$, a representation $\rho$ of $G$ on a finite-dimensional vector space $V$ all defined over $\mathbf{C}$. We call a triplet $(G, \rho, V)$ a prehomogeneous vector space (abbrev. PV) if and only if there exists an element $x \in V$ such that the orbit $\rho(G) x \subset V$ is Zariski dense in $V$. Such an element $x \in V$ is called a generic point. For example, for any triplet $(G, \rho, V)$, it is known that a triplet $\left(G \times G L_{n}, \rho \otimes \Lambda_{1}, V \otimes \mathbf{C}^{n}\right)$ is always prehomogeneous for any $n \geq m=\operatorname{dim} V$, where $\Lambda_{1}$ stands for the natural representation of $G L_{n}$ on $\mathbf{C}^{n}$. Such triplets are called trivial PVs. However, if $n<m$, the triplet $\left(G \times G L_{n}, \rho \otimes \Lambda_{1}, V \otimes \mathbf{C}^{n}\right)$ is not generally a PV. On the other hand, if the triplet $\left(G \times G L_{n}, \rho \otimes \Lambda_{1}, V \otimes \mathbf{C}^{n}\right)$ is a $\mathrm{PV}$, it is known that a triplet $\left(G \times G L_{m-n}, \rho^{*} \otimes \Lambda_{1}, V^{*} \otimes \mathbf{C}^{m-n}\right)$ is also a PV, where $\left(\rho^{*}, V^{*}\right)$ is the dual module of $(\rho, V)$. These triplets $\left(G \times G L_{n}, \rho \otimes \Lambda_{1}, V \otimes \mathbf{C}^{n}\right)$ and $\left(G \times G L_{m-n}\right.$, $\rho^{*} \otimes \Lambda_{1}, V^{*} \otimes \mathbf{C}^{m-n}$ ) are called castling transform of each other (see [7,

2010 Mathematics Subject Classification: Primary 11S90, 17 B70.

Key words and phrases: prehomogeneous vector spaces, castling transform, graded Lie algebras, Lie algebras associated with a standard quadruplet.

Received May 26, 2014.

Revised December 12, 2014. 
Definition 11, p. 39]). Now, when $G$ is reductive, we call a $\operatorname{PV}(G, \rho, V)$ a reductive PV. In [7], M. Sato and T. Kimura classified reductive PVs whose representation is irreducible by using castling transformations.

The theory of PVs is closely related to the theory of Lie algebras. The prehomogeneity condition of a triplet $(G, \rho, V)$ can be expressed by the corresponding Lie algebra and its representation $(\operatorname{Lie}(G), d \rho, V)$, where $\operatorname{Lie}(G)$ is the Lie algebra of $G$ and $d \rho$ the infinitesimal representation of $\rho$. That is, a triplet $(G, \rho, V)$ is a PV if and only if there exists an element $x \in V$ such that $d \rho(\operatorname{Lie}(G)) x=V$. This condition is useful to check the prehomogeneity of a triplet. Moreover, we can obtain PVs from a graded semisimple Lie algebra. If we let $\mathfrak{I}=\bigoplus_{n \in \mathbf{Z}} \mathfrak{l}_{n}$ be a finite-dimensional semisimple Lie algebra and $L$ the adjoint group of $\mathfrak{l}$, then the subgroup $L_{0}$ of $L$ which corresponds to $\mathfrak{I}_{0}$ acts on $\mathfrak{I}_{1}$. This representation is prehomogeneous. That is, there exists an element $x \in \mathfrak{I}_{1}$ such that $\left[\mathfrak{I}_{0}, x\right]=\mathfrak{I}_{1}$. In $[2,3,4], \mathrm{H}$. Rubenthaler studied these PVs and called them PVs of parabolic type. In other words, a PV of parabolic type is a triplet which consists of a reductive Lie algebra and its completely reducible representation which can be embedded into a finite-dimensional graded Lie algebra. Some properties of PVs of parabolic type can be described by structures of Lie algebras. For example, H. Rubenthaler classified irreducible regular PVs of parabolic type by using subalgebras of semisimple Lie algebras which are isomorphic to $\mathfrak{s l}_{2}$ (see $[2,3,4])$. However, the castling transform of a PV of parabolic type is no longer parabolic type.

In [6], the author introduced a way to embed an arbitrary finite-dimensional reductive Lie algebra $\mathfrak{g}$, its faithful and completely reducible representation $(\pi, V)$ and its dual module $\left(\pi^{*}, V^{*}\right)$ into some graded Lie algebra. For this, we use a non-degenerate symmetric and invariant bilinear form $B$ on $\mathfrak{g}$. If the quadruplet $(\mathfrak{g}, \pi, V, B)$ is a standard quadruplet (see Definition 1.6 or [6, Definition 1.9]), then we can construct a graded Lie algebra $L(\mathfrak{g}, \pi, V, B)=\bigoplus_{n \in \mathbf{Z}} V_{n}$ called the Lie algebra associated with $(\mathfrak{g}, \pi, V, B)$ and embed the above objects into it (see Theorem 1.7 or [6, Theorem 2.11]). In general, these graded Lie algebras are infinite-dimensional. By the way, H. Rubenthaler obtained a similar result in [5].

It is well-known that the Lie algebra of a reductive algebraic group is a reductive Lie algebra. Thus, by using the theory of Lie algebras associated with a standard quadruplet, any reductive $\operatorname{PV}(G, \rho, V)$ with completely reducible representation $\rho$ can be embedded into a graded Lie algebra of the form $L(\operatorname{Lie}(G), d \rho, V, B)$. Thus, it is expected that we can use the theory of Lie algebras to study a reductive PV and its castling transform in a similar way to 
the theory of PVs of parabolic type. However, since we have the Lie algebra $L(\operatorname{Lie}(G), d \rho, V, B)$ regardless whether $(G, \rho, V)$ is a $\mathrm{PV}$ or not, it is required to give a Lie algebraic property of $L(\operatorname{Lie}(G), d \rho, V, B)$ which corresponds to the prehomogeneity of the triplet $(G, \rho, V)$. The purpose of this paper is to answer this problem, i.e., we shall give a necessary and sufficient condition for a triplet $(G, \rho, V)$ to be a PV by using the structure of the graded Lie algebra $L(\operatorname{Lie}(G), d \rho, V, B)$. Moreover, we give another proof of castling transformation as an application.

This paper consists of two sections. In section 1, we consider about the $\Phi$-map of quadruplets. First, we give the definition of $\Phi$-map (Definition 1.1) and some examples (Example 1.2, 1.3, 1.4). Next, we give the $\Phi$-maps of a direct sum and a tensor product of modules (Proposition 1.5). At the end of this section, we introduce the notion of the Lie algebras associated with a standard quadruplet (Definition 1.6 and Theorem 1.7).

In section 2, we shall define the notion of prehomogeneous quadruplet by using $\Phi$-map. The prehomogeneity of quadruplets corresponds to the prehomogeneity of triplets. For this, we shall give a way to describe the prehomogeneity condition of $(G, \rho, V)$ by using the $\Phi$-map of $(\operatorname{Lie}(G), d \rho, V, B)$ (Theorem 2.1). Moreover, if $(\operatorname{Lie}(G), d \rho, V, B)$ is a standard quadruplet, we can describe the prehomogeneity of $(G, \rho, V)$ by the restricted bracket product $[\cdot, \cdot]: V_{-1} \times V_{1} \rightarrow V_{0}$ of the graded Lie algebra $L(\operatorname{Lie}(G), d \rho, V, B)=\bigoplus_{n \in \mathbf{Z}} V_{n}$ (Theorem 2.4). A prehomogeneous quadruplet is a quadruplet such that its $\Phi$-map satisfies the same condition.

In the remaining part of this section, we shall consider the prehomogeneity of triplets of the form $\left(G \times G L_{n}, \rho \otimes \Lambda_{1}, V \otimes \mathbf{C}^{n}\right)$. It can be described by the $\Phi$-map of $(\operatorname{Lie}(G), d \rho, V, B)$. That is, in general, the prehomogeneity condition of a quadruplet of the form $\left(\mathfrak{g} \oplus \mathfrak{g l}_{n}, \pi \otimes \Lambda_{1}, V \otimes \mathbf{C}^{n}, B \oplus T_{n}\right)$, where $T_{n}$ is a bilinear form on $\mathfrak{g l}_{n}$ (see (1.3)), can be described by the $\Phi$-map of $(\mathfrak{g}, \pi, V, B)$ (Lemma 2.7). Finally, as an application of this lemma, we shall give another proof of castling transformation by using the Lie algebraic calculation (Theorem 2.10). In particular, the correspondence of generic points of $\left(G \times G L_{n}, \rho \otimes \Lambda_{1}, V \otimes \mathbf{C}^{n}\right)$ and one of $\left(G \times G L_{m-n}, \rho^{*} \otimes \Lambda_{1}, V^{*} \otimes \mathbf{C}^{m-n}\right)$ and their generic isotropy subalgebras can be described by using the Lie algebraic calculation.

Notation: We denote the space of matrices of size $n \times m$ by $M(n, m ; \mathbf{C})$, the trace of a matrix $A$ by $\operatorname{Tr}(A)$, the transpose of a matrix $X$ by ${ }^{\mathrm{t}} X$, the zero matrix and the unit matrix of size $n \times n$ by $0_{n}$ and $1_{n}$. In this paper, all objects are defined over $\mathbf{C}$. 


\section{The Ф-Map of a Quadruplet}

Let $\mathfrak{g}$ be a finite-dimensional reductive Lie algebra and $\pi$ a representation of $\mathfrak{g}$ on a finite-dimensional vector space $V$. Moreover, we denote the dual module of $(\pi, V)$ by $\left(\pi^{*}, V^{*}\right)$ and the pairing between $V$ and $V^{*}$ by $\langle\cdot, \cdot\rangle$. Now, by the theory of Lie algebras, it is known that there exists a non-degenerate symmetric and invariant bilinear form $B$ on $\mathfrak{g}$ (see [1, Chapter $1 \S 6.4$ Proposition 5]). Throughout this paper, we use these notation. Then we can define the following linear map from the quadruplet $(\mathfrak{g}, \pi, V, B)$ which plays important roles in this paper.

Definition 1.1 ( $\Phi$-map of a quadruplet). We define a linear map $\Phi_{\pi}$ from $V \otimes V^{*}$ to $\mathfrak{g}$ by the following equation:

$$
B\left(a, \Phi_{\pi}(v \otimes \phi)\right)=\langle\pi(a) v, \phi\rangle=-\left\langle v, \pi^{*}(a) \phi\right\rangle
$$

for any $a \in \mathfrak{g}, v \in V$ and $\phi \in V^{*}$. Since $B$ is non-degenerate, the equation (1.1) determines the linear map $\Phi_{\pi}$ uniquely. We call this map $\Phi_{\pi}$ the $\Phi$-map of $(\mathfrak{g}, \pi, V, B)$ (see [6, Definition 1.1]). Moreover, for an element $v \in V$, we can define a linear map $\Phi_{\pi, v}$ from $V^{*}$ to $\mathfrak{g}$ by:

$$
\Phi_{\pi, v}(\phi):=\Phi_{\pi}(v \otimes \phi)
$$

where $\phi \in V^{*}$. We call this map $\Phi_{\pi, v}$ the $\Phi$-map at $v$ of $(\mathfrak{g}, \pi, V, B)$.

Example 1.2. Let $(\mathfrak{g}, \pi, V, B)$ be a quadruplet $\left(\mathfrak{g l}_{n}, \Lambda_{1}, \mathbf{C}^{n}, T_{n}\right)$, where $\Lambda_{1}$ is the natural representation of $\mathrm{gl}_{n}$ on the space of column vectors $\mathbf{C}^{n}=M(n, 1 ; \mathbf{C})$ and $T_{n}$ is a bilinear form on $\mathrm{gl}_{n}$ defined by

$$
T_{n}\left(a, a^{\prime}\right):=\operatorname{Tr}\left(a a^{\prime}\right)
$$

where $a, a^{\prime} \in \mathfrak{g}$. Then the dual space $V^{*}$ can be identified with $\mathbf{C}^{n}$. The representation $\Lambda_{1}^{*}$ and the pairing $\langle\cdot, \cdot\rangle$ are given as follows:

$$
\begin{aligned}
& \Lambda_{1}^{*}(a) \phi:=-{ }^{\mathrm{t}} a \phi, \\
& \langle v, \phi\rangle:={ }^{\mathrm{t}} v \phi
\end{aligned}
$$

where $a \in \mathfrak{g}, v \in V$ and $\phi \in V^{*}$. Then the $\Phi$-map is given as:

$$
\Phi_{\Lambda_{1}}(v \otimes \phi)=v^{\mathrm{t}} \phi
$$


EXAMPLE 1.3. Let $(\mathfrak{g}, \pi, V, B)$ be a quadruplet $\left(\mathfrak{s o}_{n}, \Lambda_{1}, \mathbf{C}^{n},\left.T_{n}\right|_{\mathfrak{s o}_{n} \times \mathfrak{s o}_{n}}\right)$, where $\Lambda_{1}$ is the natural representation of $\mathfrak{s i}_{n}$ on $\mathbf{C}^{n}=M(n, 1 ; \mathbf{C})$. Then the dual space $V^{*}$ can be identified with $\mathbf{C}^{n}$. The representation $\Lambda_{1}^{*}$ and the pairing $\langle\cdot, \cdot\rangle$ are given as follows:

$$
\begin{gathered}
\Lambda_{1}^{*}(a) \phi:=a \phi, \\
\langle v, \phi\rangle:={ }^{\mathrm{t}} v \phi
\end{gathered}
$$

where $a \in \mathfrak{g}, v \in V$ and $\phi \in V^{*}$. Then the $\Phi$-map is given as:

$$
\Phi_{\Lambda_{1}}(v \otimes \phi)=\frac{1}{2}\left(v^{\mathrm{t}} \phi-\phi^{\mathrm{t}} v\right) .
$$

EXAMPLe 1.4. Let $(\mathfrak{g}, \pi, V, B)$ be a quadruplet $\left(\mathfrak{s p}_{n}, \Lambda_{1}, \mathbf{C}^{2 n},\left.T_{2 n}\right|_{\mathfrak{s p}_{n} \times \mathfrak{s p}_{n}}\right)$, where $\Lambda_{1}$ is the natural representation of $\mathfrak{s p}_{n}=\left\{X \in \mathfrak{g l}_{2 n} \mid{ }^{\mathrm{t}} X J_{n}+J_{n} X=0\right.$, $\left.J_{n}:=\left(\begin{array}{cc}0_{n} & 1_{n} \\ -1_{n} & 0_{n}\end{array}\right)\right\}$ on $\mathbf{C}^{2 n}=M(2 n, 1 ; \mathbf{C})$. Then the dual space $V^{*}$ can be identified with $\mathbf{C}^{2 n}$. The representation $\Lambda_{1}^{*}$ and the pairing $\langle\cdot, \cdot\rangle$ are given as follows:

$$
\begin{aligned}
& \Lambda_{1}^{*}(a) \phi:=a \phi, \\
& \langle v, \phi\rangle:={ }^{\mathrm{t}} v J_{n} \phi
\end{aligned}
$$

where $a \in \mathfrak{g}, v \in V$ and $\phi \in V^{*}$. Then the $\Phi$-map is given as:

$$
\Phi_{\Lambda_{1}}(v \otimes \phi)=-\frac{1}{2}\left(v^{\mathrm{t}} \phi J_{n}+\phi^{\mathrm{t}} v J_{n}\right)
$$

The $\Phi$-map of a direct sum or tensor product of modules can be obtained as follows.

Proposition 1.5. Let $\mathfrak{g}$ be any reductive Lie algebra, $\pi_{i}, \sigma_{i}$ representations of $\mathfrak{g}$ on $V_{i}$ and $U_{i}(i=1,2)$ and $B$ a non-degenerate symmetric and invariant bilinear form on g. Then for quadruplets $\left(\mathfrak{g}, \pi_{1} \oplus \pi_{2}, V_{1} \oplus V_{2}, B\right)$ and $\left(\mathfrak{g}, \sigma_{1} \otimes \sigma_{2}, U_{1} \otimes U_{2}\right.$, $B)$, their $\Phi$-maps $\Phi_{\pi_{1} \oplus \pi_{2}}$ and $\Phi_{\sigma_{1} \otimes \sigma_{2}}$ are given as follows:

$$
\begin{aligned}
& \Phi_{\pi_{1} \oplus \pi_{2}}\left(\left(v_{1}, v_{2}\right) \otimes\left(\phi_{1}, \phi_{2}\right)\right)=\Phi_{\pi_{1}}\left(v_{1} \otimes \phi_{1}\right)+\Phi_{\pi_{2}}\left(v_{2} \otimes \phi_{2}\right), \\
& \Phi_{\sigma_{1} \otimes \sigma_{2}}\left(\left(u_{1} \otimes u_{2}\right) \otimes\left(\psi_{1} \otimes \psi_{2}\right)\right) \\
& \quad=\left\langle u_{2}, \psi_{2}\right\rangle \Phi_{\sigma_{1}}\left(u_{1} \otimes \psi_{1}\right)+\left\langle u_{1}, \psi_{1}\right\rangle \Phi_{\sigma_{2}}\left(u_{2} \otimes \psi_{2}\right)
\end{aligned}
$$


where $v_{i} \in V_{i}, u_{i} \in U_{i}, \phi_{i} \in V_{i}^{*}, \psi_{i} \in U_{i}^{*}$ and $\Phi_{\pi_{i}}, \Phi_{\sigma_{i}}$ are the $\Phi$-maps of the quadruplets $\left(\mathfrak{g}, \pi_{i}, V_{i}, B\right)$ and $\left(\mathfrak{g}, \sigma_{i}, U_{i}, B\right)(i=1,2)$.

Proof. The dual modules of $V_{1} \oplus V_{2}$ and $U_{1} \otimes U_{2}$ can be identified with $V_{1}^{*} \oplus V_{2}^{*}$ and $U_{1}^{*} \otimes U_{2}^{*}$ respectively. The pairings between them are given as follows:

$$
\begin{aligned}
& \left\langle\left(v_{1}, v_{2}\right),\left(\phi_{1}, \phi_{2}\right)\right\rangle:=\left\langle v_{1}, \phi_{1}\right\rangle+\left\langle v_{2}, \phi_{2}\right\rangle, \\
& \left\langle u_{1} \otimes u_{2}, \psi_{1} \otimes \psi_{2}\right\rangle:=\left\langle u_{1}, \psi_{1}\right\rangle\left\langle u_{2}, \psi_{2}\right\rangle .
\end{aligned}
$$

Then, our claim can be checked by a direct calculation.

When a quadruplet $(\mathfrak{g}, \pi, V, B)$ is a standard quadruplet, its $\Phi$-map is closely related to the bracket product of the Lie algebra associated with $(\mathfrak{g}, \pi, V, B)$ (see [6, Definition 1.9 and Theorem 2.11]).

Definition 1.6 (Standard quadruplets). If a quadruplet $(\mathfrak{g}, \pi, V, B)$ satisfies the following conditions, then we call it a standard quadruplet:

$$
\begin{aligned}
& \pi \text { is faithful, } \\
& \pi \text { is completely reducible, } \\
& \{v \in V \mid \pi(a) v=0 \text { for all } a \in \mathfrak{g}\}=\{0\} .
\end{aligned}
$$

THEOREM 1.7 (Lie algebra associated with a standard quadruplet). For $a$ standard quadruplet $(\mathfrak{g}, \pi, V, B)$, there exists a graded Lie algebra $L(\mathfrak{g}, \pi, V, B)=$ $\bigoplus_{n \in \mathbf{Z}} V_{n}$ called the Lie algebra associated with $(\mathfrak{g}, \pi, V, B)$. This Lie algebra satisfies that $V_{0}, V_{1}, V_{-1}$ are isomorphic to $\mathfrak{g}, V, V^{*}$ respectively. The restriction of the bracket product of $L(\mathfrak{g}, \pi, V, B)$ to $V_{1} \times V_{-1} \rightarrow V_{0}$ can be identified with the $\Phi$-map of $(\mathfrak{g}, \pi, V, B)$.

\section{Prehomogeneous Vector Spaces}

\subsection{Prehomogeneous Quadruplets}

Recall that a triplet $(G, \rho, V)$ is called a prehomogeneous vector space (abbrev. PV) if and only if there exists an element $x \in V$ such that $\rho(G) x$ is Zariski dense in $V$. In this section, we shall introduce the notion of "prehomogeneity" of quadruplets which corresponds to PVs. For this, we give the 
following theorem to describe the prehomogeneity of $(G, \rho, V)$ by using the $\Phi$-map of a quadruplet of the form $(\operatorname{Lie}(G), d \rho, V, B)$.

THeOREM 2.1. Let $G$ be a connected reductive algebraic group, $V$ a finitedimensional vector space and $\rho$ a representation of $G$ on $V$. Let $\operatorname{Lie}(G)$ be the Lie algebra of $G$ and $d \rho$ the infinitesimal representation of $\rho$ on $V$. Then the triplet $(G, \rho, V)$ is prehomogeneous if and only if there exists an element $x \in V$ and a nondegenerate symmetric and invariant bilinear form $B$ on $\operatorname{Lie}(G)$ such that the $\Phi$-map at $x$ of the quadruplet $(\operatorname{Lie}(G), d \rho, V, B)$, denoted by $\Phi_{d \rho, x}: V^{*} \rightarrow \operatorname{Lie}(G)$, is injective.

Proof. Assume that $(G, \rho, V)$ is a $\mathrm{PV}$ and $x$ is its generic point. Then it is known that the vector subspace $d \rho(\operatorname{Lie}(G)) x$ of $V$ coincides with $V$ (see [7, Proposition 2, p. 36]). Let us show that the $\Phi$-map at $x$ denoted by $\Phi_{d \rho, x}$ is injective. In fact, if $\Phi_{d \rho, x}$ is not injective, there exists a non-zero element $\phi \in V^{*}$ such that $\Phi_{d \rho, x}(\phi)=0$ and we have

$$
0=B\left(a, \Phi_{d \rho, x}(\phi)\right)=\langle d \rho(a) x, \phi\rangle
$$

for any $a \in \operatorname{Lie}(G)$. This is a contradiction to the assumption that $d \rho(\operatorname{Lie}(G)) x$ $=V$. Thus $\Phi_{d \rho, x}$ is injective.

Conversely, suppose that there exists a non-degenerate symmetric invariant bilinear form $B$ on $\operatorname{Lie}(G)$ and an element $x \in V$ such that the linear map $\Phi_{d \rho, x}$ is injective. Then $(G, \rho, V)$ is a PV and $x$ is its generic point. Indeed, if $x$ is not a generic point, then $d \rho(\operatorname{Lie}(G)) x$ is a proper subspace of $V$ and thus there exists a non-zero element $\phi \in V^{*}$ such that $0=\langle d \rho(a) x, \phi\rangle=B\left(a, \Phi_{d \rho, x}(\phi)\right)$ for any $a \in \operatorname{Lie}(G)$. Since $B$ is non-degenerate, we have $\Phi_{d \rho, x}(\phi)=0$. This is a contradiction to the assumption that $\Phi_{d \rho, x}$ is injective. Therefore we have $d \rho(\operatorname{Lie}(G)) x$ $=V$ and thus $x$ is a generic point.

Definition 2.2. Let $\mathfrak{g}$ be a finite-dimensional reductive Lie algebra, $\pi$ a representation of $\mathfrak{g}$ on a finite-dimensional vector space $V$ and $B$ a non-degenerate symmetric invariant bilinear form on $\mathfrak{g}$. We say that a quadruplet $(\mathfrak{g}, \pi, V, B)$ is a prehomogeneous quadruplet if and only if there exists an element $x \in V$ such that $\Phi_{\pi, x}: V^{*} \rightarrow \mathfrak{g}$ is injective. We call such an element $x \in V$ a generic point of $(\mathfrak{g}, \pi, V, B)$. A generic point $x$ satisfies $\pi(\mathfrak{g}) x=V$.

REMARK 2.3. Note that if $(\mathfrak{g}, \pi, V, B)$ is prehomogeneous, then for any other non-degenerate symmetric and invariant bilinear form $B^{\prime}$ on $\mathfrak{g},\left(\mathfrak{g}, \pi, V, B^{\prime}\right)$ is also 
prehomogeneous with the same generic points, i.e. the prehomogeneity of a quadruplet is independent to the choice of a bilinear form. It can be checked by the proof of Proposition 2.1.

In particular, when a quadruplet $(\mathfrak{g}, \pi, V, B)$ is standard, its prehomogeneity is described in terms of graded Lie algebras. We can obtain the following theorem from Theorems 1.7 and 2.1 .

THEOREM 2.4. Under the notation of Definition 2.2, we assume that $(\mathfrak{g}, \pi, V, B)$ is a standard quadruplet. Then the quadruplet $(\mathfrak{g}, \pi, V, B)$ is prehomogeneous if and only if there exists an element $x \in V_{1}$ such that

$$
\left.(\operatorname{ad} x)\right|_{V_{-1}}: V_{-1} \rightarrow V_{0}
$$

is injective, where ad stands for the adjoint representation of $L(\mathfrak{g}, \pi, V, B)=$ $\bigoplus_{n \in \mathbf{Z}} V_{n}$ on itself.

To consider a $\mathrm{PV}(G, \rho, V)$ whose representation $\rho$ is completely reducible, we can assume that $\rho$ is faithful without loss of generality. Then a quadruplet $(\operatorname{Lie}(G), d \rho, V, B)$ is a standard quadruplet for any non-degenerate symmetric invariant bilinear form $B$. Thus, we can say that any reductive PV with completely reducible representation can be embedded into some graded Lie algebra which corresponds to a prehomogeneous standard quadruplet.

Example 2.5. A triplet $\left(S O_{n}, \Lambda_{1}, \mathbf{C}^{n}\right)$, where $\Lambda_{1}$ is the natural representation of $S O_{n}$ on $\mathbf{C}^{n}=M(n, 1 ; \mathbf{C})$, is not a PV for any $n \geq 3$. To check this, under the notation of Example 1.3, let us show that the quadruplet $\left(\mathfrak{s o}_{n}, \Lambda_{1}, \mathbf{C}^{n},\left.T_{n}\right|_{\mathfrak{s o}_{n} \times \mathfrak{s o}_{n}}\right)$ is not prehomogeneous. In fact, for any column vector $v \in \mathbf{C}^{n}$, we have

$$
\Phi_{\Lambda_{1}, v}(v)=\frac{1}{2}\left(v^{\mathrm{t}} v-v^{\mathrm{t}} v\right)=0
$$

Thus we have our claim.

However, a quadruplet $\left(\mathrm{gl}_{1} \oplus \mathfrak{s o}_{n}, \square \otimes \Lambda_{1}, \mathbf{C} \otimes \mathbf{C}^{n}, B\right)$ is prehomogeneous, where $\square$ is a scalar multiplication of $\mathrm{gl}_{1}=\mathbf{C}$ and $B$ is a bilinear form defined by:

$$
B\left((a, A),\left(a^{\prime}, A^{\prime}\right)\right):=-a a^{\prime}+\frac{1}{2} \operatorname{Tr}\left(A A^{\prime}\right)
$$

where $a, a^{\prime} \in \mathfrak{g l}_{1}=\mathbf{C}$ and $A, A^{\prime} \in \mathfrak{s o}_{n}$. In fact, identifying $\mathbf{C} \otimes \mathbf{C}^{n}$ with $\mathbf{C}^{n}$, the representation $\square \otimes \Lambda_{1}$ and the map $\Phi_{\square \otimes \Lambda_{1}}$ are given as follows: 


$$
\begin{aligned}
& \left(\square \otimes \Lambda_{1}\right)(a, A) \cdot v=a v+A v, \\
& \Phi_{\square \otimes \Lambda_{1}}(v \otimes \phi)=\left(-{ }^{\mathrm{t}} v \phi, v^{\mathrm{t}} \phi-\phi^{\mathrm{t}} v\right) .
\end{aligned}
$$

Put $v_{0}:={ }^{\mathrm{t}}(1,0, \ldots, 0)$. Then we have

$$
\left(\square \otimes \Lambda_{1}\right)\left(\Phi_{\square \otimes \Lambda_{1}, v_{0}}(\phi)\right) \cdot v_{0}=-\phi
$$

for any $\phi \in V^{*}$. Thus the map $\Phi_{\square \otimes \Lambda_{1}, v_{0}}$ is injective. Therefore $\left(\mathfrak{g l}_{1} \oplus \mathfrak{s o}_{n}\right.$, $\left.\square \otimes \Lambda_{1}, \mathbf{C} \otimes \mathbf{C}^{n}, B\right)$ is a prehomogeneous quadruplet.

\subsection{Triplets of the Form $\left(G \times G L_{n}, \rho \otimes \Lambda_{1}, V \otimes \mathbf{C}^{n}\right)$}

In this section, we shall consider an important theorem in the theory of prehomogeneous vector spaces, castling transformation. We shall study triplets of the form $\left(G \times G L_{n}, \rho \otimes \Lambda_{1}, V \otimes \mathbf{C}^{n}\right)$ by using quadruplets of the form $\left(\mathfrak{g} \oplus \mathfrak{g l}_{n}, \pi \otimes \Lambda_{1}, V \otimes \mathbf{C}^{n}, B \oplus T_{n}\right)$, where $B \oplus T_{n}$ is a bilinear form on $\mathfrak{g} \oplus \mathfrak{g l}_{n}$ defined by $\left(B \oplus T_{n}\right)\left((a, A),\left(a^{\prime}, A^{\prime}\right)\right):=B\left(a, a^{\prime}\right)+T_{n}\left(A, A^{\prime}\right) \quad\left(a, a^{\prime} \in \mathfrak{g}, A, A^{\prime} \in \mathfrak{g l}_{n}\right)$. To consider the prehomogeneity condition of them, we give the following definition.

Definition 2.6. Let $\mathfrak{g}$ be a reductive Lie algebra, $\pi$ a representation of $\mathfrak{g}$ on a finite-dimensional vector space $V, B$ a non-degenerate symmetric invariant bilinear form on $\mathfrak{g}$. For the quadruplet $(\mathfrak{g}, \pi, V, B)$, any $n \in \mathbf{N}$ and $n$-vectors $v_{1}, \ldots, v_{n} \in V$ and $\phi_{1}, \ldots, \phi_{n} \in V^{*}$, we define vector subspaces $S_{\left(v_{1}, \ldots, v_{n}\right)} \subset\left(V^{*}\right)^{n}$ and $S_{\left(\phi_{1}, \ldots, \phi_{n}\right)}^{*} \subset V^{n}$ as follows:

$$
\begin{aligned}
& S_{\left(v_{1}, \ldots, v_{n}\right)}:=\left\{\left(\psi_{1}, \ldots, \psi_{n}\right) \in\left(V^{*}\right)^{n} \mid \sum_{l=1}^{n} \Phi_{\pi}\left(v_{l} \otimes \psi_{l}\right)=0,\left\langle v_{i}, \psi_{j}\right\rangle=0\right. \\
&\text { for } 1 \leq i, j \leq n\}, \\
& S_{\left(\phi_{1}, \ldots, \phi_{n}\right)}^{*}:=\left\{\left(u_{1}, \ldots, u_{n}\right) \in V^{n} \mid \sum_{l=1}^{n} \Phi_{\pi}\left(u_{l} \otimes \phi_{l}\right)=0,\left\langle u_{i}, \phi_{j}\right\rangle=0\right. \\
&\text { for } 1 \leq i, j \leq n\}
\end{aligned}
$$

where $\Phi_{\pi}$ is the $\Phi$-map of $(\mathfrak{g}, \pi, V, B)$. 
Lemma 2.7. We continue to use the notation of Example 1.2 and Definition 2.6. Then a quadruplet $\left(\mathfrak{g} \oplus \mathfrak{g l}_{n}, \pi \otimes \Lambda_{1}, V \otimes \mathbf{C}^{n}, B \oplus T_{n}\right)$ (respectively $\left(\mathfrak{g} \oplus \mathfrak{g l}_{n}\right.$, $\left.\left.\pi^{*} \otimes \Lambda_{1}, V^{*} \otimes \mathbf{C}^{n}, B \oplus T_{n}\right)\right)$ is prehomogeneous if and only if there exists $n$-vectors $v_{1}, \ldots, v_{n} \in V$ (respectively $\left.\phi_{1}, \ldots, \phi_{n} \in V^{*}\right)$ such that $S_{\left(v_{1}, \ldots, v_{n}\right)}=\{(0, \ldots, 0)\}$ (respectively $\left.S_{\left(\phi_{1}, \ldots, \phi_{n}\right)}^{*}=\{(0, \ldots, 0)\}\right)$.

Proof. We prove for a quadruplet $\left(\mathfrak{g} \oplus \mathfrak{g l}_{n}, \pi \otimes \Lambda_{1}, V \otimes \mathbf{C}^{n}, B \oplus T_{n}\right)$. For a quadruplet $\left(\mathfrak{g} \oplus \mathfrak{g l}_{n}, \pi^{*} \otimes \Lambda_{1}, V^{*} \otimes \mathbf{C}^{n}, B \oplus T_{n}\right)$, our claim can be proved by the same way.

Let $e_{i} \in \mathbf{C}^{n}$ be the column vector whose coefficients are all zero except the $i$-th one which is equal to 1 and $E_{i j} \in \mathfrak{g l}_{n}$ be the $n \times n$ matrix whose coefficients are all zero except the $(i, j)$-th one which is equal to 1 . Then for any $v \in V$ and $\phi \in V^{*}$, we have

$$
\Phi_{\pi \otimes \Lambda_{1}}\left(\left(v \otimes e_{i}\right) \otimes\left(\phi \otimes e_{j}\right)\right)=\left(\delta_{i j} \Phi_{\pi}(v \otimes \phi),\langle v, \phi\rangle E_{i j}\right)
$$

from Example 1.2 and Proposition 1.5 where $\delta_{i j}$ is the Kronecker delta.

Suppose that $\left(\mathfrak{g} \oplus \mathfrak{g l}_{n}, \pi \otimes \Lambda_{1}, V \otimes \mathbf{C}^{n}, B \oplus T_{n}\right)$ is a prehomogeneous quadruplet and $v_{1} \otimes e_{1}+\cdots+v_{n} \otimes e_{n} \in V \otimes \mathbf{C}^{n}$ is its generic point. Then we have $S_{\left(v_{1}, \ldots, v_{n}\right)}=\{(0, \ldots, 0)\}$. In fact, take an arbitrary element $\left(\psi_{1}, \ldots, \psi_{n}\right) \in S_{\left(v_{1}, \ldots, v_{n}\right)}$, then we have:

$$
\begin{aligned}
\Phi_{\pi \otimes \Lambda_{1}} & \left(\left(\sum_{1 \leq i \leq n} v_{i} \otimes e_{i}\right) \otimes\left(\sum_{1 \leq j \leq n} \psi_{j} \otimes e_{j}\right)\right) \\
& =\left(\Phi_{\pi}\left(\sum_{1 \leq k \leq n} v_{k} \otimes \psi_{k}\right), \sum_{1 \leq i, j \leq n}\left\langle v_{i}, \psi_{j}\right\rangle E_{i j}\right)=0 .
\end{aligned}
$$

Therefore, we have $\psi_{1} \otimes e_{1}+\cdots+\psi_{n} \otimes e_{n}=0$ and thus $\left(\psi_{1}, \ldots, \psi_{n}\right)=(0, \ldots, 0)$.

Conversely, assume that $n$-vectors $v_{1}, \ldots, v_{n} \in V$ satisfy $S_{\left(v_{1}, \ldots, v_{n}\right)}=$ $\{(0, \ldots, 0)\}$. Then $v_{1} \otimes e_{1}+\cdots+v_{n} \otimes e_{n}$ is a generic point of $(\mathfrak{g}, \pi, V, B)$. In fact, suppose that $\phi_{1} \otimes e_{1}+\cdots+\phi_{n} \otimes e_{n}$ satisfies

$$
\Phi_{\pi \otimes \Lambda_{1}}\left(\left(\sum_{1 \leq i \leq n} v_{i} \otimes e_{i}\right) \otimes\left(\sum_{1 \leq j \leq n} \phi_{j} \otimes e_{j}\right)\right)=0,
$$

then we can obtain that $\left(\phi_{1}, \ldots, \phi_{n}\right) \in S_{\left(v_{1}, \ldots, v_{n}\right)}$ and thus $\phi_{1} \otimes e_{1}+\cdots+\phi_{n} \otimes e_{n}=$ 0 . This completes the proof.

The following corollary is immediate. 
COROLlary 2.8. Under the notation of Example 1.2 and Definition 2.6, if we let $n \geq m=\operatorname{dim} V$, then a quadruplet $\left(\mathfrak{g} \oplus \mathfrak{g l}_{n}, \pi \otimes \Lambda_{1}, V \otimes \mathbf{C}^{n}, B \oplus T_{n}\right)$ is prehomogeneous. Such quadruplets correspond to triplets which are called trivial PVS (see [7, Definition 5, p. 43]).

EXAMPLE 2.9. It is known that a triplet $\left(G L_{3} \times G L_{3} \times G L_{2}, \Lambda_{1} \otimes \Lambda_{1} \otimes \Lambda_{1}\right.$, $\mathbf{C}^{3} \otimes \mathbf{C}^{3} \otimes \mathbf{C}^{2}$ ) is a PV (see [7, Proposition 16, p. 100]). Thus the corresponding quadruplet $\left(\mathfrak{g l}_{3} \oplus \mathfrak{g l}_{3} \oplus \mathfrak{g l}_{2}, \Lambda_{1} \otimes \Lambda_{1} \otimes \Lambda_{1}, \mathbf{C}^{3} \otimes \mathbf{C}^{3} \otimes \mathbf{C}^{2}, T_{3} \oplus T_{3} \oplus T_{2}\right)$ is prehomogeneous. Let us check this by using Lemma 2.7 .

First, let us consider a quadruplet $(\mathfrak{g}, \rho, V, B):=\left(\mathfrak{g l}_{3} \oplus \mathfrak{g l}_{3}, \Lambda_{1} \otimes \Lambda_{1}\right.$, $\left.\mathbf{C}^{3} \otimes \mathbf{C}^{3}, T_{3} \oplus T_{3}\right)$. The representation $\pi=\Lambda_{1} \otimes \Lambda_{1}$ can be identified with the representation of $\mathfrak{g l}_{3} \oplus \mathfrak{g l}_{3}$ on the space of square matrices of size 3 defined by

$$
\left(\Lambda_{1} \otimes \Lambda_{1}\right)(A, B) \cdot X:=A X+X^{\mathrm{t}} B
$$

where $(A, B) \in \mathfrak{g}=\mathfrak{g l}_{3} \oplus \mathfrak{g l}_{3}$ and $X \in V=M(3,3 ; \mathbf{C})$. Then the dual module $V^{*}$ is also identified with $M(3,3 ; \mathbf{C})$. The dual representation $\pi^{*}$, the pairing $\langle\cdot, \cdot\rangle$ between $V$ and $V^{*}$ and the $\Phi$-map of the quadruplet $(\mathfrak{g}, \pi, V, B)$ are given by

$$
\begin{aligned}
& \left(\Lambda_{1} \otimes \Lambda_{1}\right)^{*}(A, B) \cdot \xi:=-{ }^{\mathrm{t}} A \xi-\xi B, \\
& \langle X, \xi\rangle:=\operatorname{Tr}\left(X^{\mathrm{t}} \xi\right), \\
& \Phi_{\Lambda_{1} \otimes \Lambda_{1}}(X \otimes \xi)=\left(X^{\mathrm{t}} \xi,{ }^{\mathrm{t}} X \xi\right)
\end{aligned}
$$

where $(A, B) \in \mathfrak{g}, X \in V$ and $\xi \in V^{*}=M(3,3 ; \mathbf{C})$.

Next, let us show that the following matrices

$$
X_{1}:=\left(\begin{array}{ccc}
1 & 0 & 0 \\
0 & 1 & 0 \\
0 & 0 & 1
\end{array}\right), \quad X_{2}:=\left(\begin{array}{ccc}
1 & 0 & 0 \\
0 & -1 & 0 \\
0 & 0 & 0
\end{array}\right) \in V
$$

satisfy $S_{\left(X_{1}, X_{2}\right)}=\{(0,0)\} \subset\left(V^{*}\right)^{2}$. In fact, the orthogonal space $\left(\mathbf{C} X_{1}+\mathbf{C} X_{2}\right)^{\perp} \subset$ $V^{*}$ is given by

$$
\left(\mathbf{C} X_{1}+\mathbf{C} X_{2}\right)^{\perp}=\left\{\left(\begin{array}{ccc}
a & b & e \\
c & a & f \\
g & h & -2 a
\end{array}\right) \mid a, \ldots, h \in \mathbf{C}\right\} .
$$

We take arbitrary elements

$$
\xi_{i}:=\left(\begin{array}{ccc}
a_{i} & b_{i} & e_{i} \\
c_{i} & a_{i} & f_{i} \\
g_{i} & h_{i} & -2 a_{i}
\end{array}\right) \in\left(\mathbf{C} X_{1}+\mathbf{C} X_{2}\right)^{\perp} \quad(i=1,2) .
$$


Then we have

$$
\begin{aligned}
\Phi_{\Lambda_{1} \otimes \Lambda_{1}}\left(X_{1} \otimes \xi_{1}+X_{2} \otimes \xi_{2}\right) & \\
= & \left(\left(\begin{array}{ccc}
a_{1}+a_{2} & c_{1}+c_{2} & g_{1}+g_{2} \\
b_{1}-b_{2} & a_{1}-a_{2} & h_{1}-h_{2} \\
e_{1} & f_{1} & -2 a_{1}
\end{array}\right),\left(\begin{array}{ccc}
a_{1}+a_{2} & b_{1}+b_{2} & e_{1}+e_{2} \\
c_{1}-c_{2} & a_{1}-a_{2} & f_{1}-f_{2} \\
g_{1} & h_{1} & -2 a_{1}
\end{array}\right)\right)
\end{aligned}
$$

$\in \mathfrak{g}$.

Thus, by an easy calculation, we can obtain that $\Phi_{\Lambda_{1} \otimes \Lambda_{1}}\left(X_{1} \otimes \xi_{1}+X_{2} \otimes \xi_{2}\right)=0$ if and only if $\xi_{1}=\xi_{2}=0 \in V^{*}$. Therefore we obtain that a quadruplet $\left(\mathrm{gl}_{3} \oplus\right.$ $\left.\mathfrak{g l}_{3} \oplus \mathfrak{g l}_{2}, \Lambda_{1} \otimes \Lambda_{1} \otimes \Lambda_{1}, \mathbf{C}^{3} \otimes \mathbf{C}^{3} \otimes \mathbf{C}^{2}, T_{3} \oplus T_{3} \oplus T_{2}\right)$ is prehomogeneous.

As an application of Lemma 2.7, we can obtain another proof of castling transformation. For this, let us show the following theorem about quadruplets.

THEOREM 2.10 (Castling transformation). Under the notation of Example 1.2 and Definition 2.6, we let $n<m=\operatorname{dim} V$. Then a quadruplet $\left(\mathfrak{g} \oplus \mathfrak{g l}_{n}, \pi \otimes \Lambda_{1}\right.$, $\left.V \otimes \mathbf{C}^{n}, B \oplus T_{n}\right)$ is prehomogeneous if and only if a quadruplet $\left(\mathfrak{g} \oplus \mathfrak{g l}_{m-n}\right.$, $\left.\pi^{*} \otimes \Lambda_{1}, V^{*} \otimes \mathbf{C}^{m-n}, B \oplus T_{m-n}\right)$ is also prehomogeneous. Moreover, if $\left(\mathfrak{g} \oplus \mathfrak{g l}_{n}\right.$, $\left.\pi \otimes \Lambda_{1}, V \otimes \mathbf{C}^{n}, B \oplus T_{n}\right) \quad$ is prehomogeneous (and thus $\quad\left(\mathfrak{g} \oplus \mathfrak{g l}_{m-n}, \pi^{*} \otimes \Lambda_{1}\right.$, $\left.V^{*} \otimes \mathbf{C}^{m-n}, B \oplus T_{m-n}\right)$ is also prehomogeneous), then for any generic point $x \in$ $V \otimes \mathbf{C}^{n}$ of $\left(\mathfrak{g} \oplus \mathfrak{g l}_{n}, \pi \otimes \Lambda_{1}, V \otimes \mathbf{C}^{n}, B \oplus T_{n}\right)$ there exists a generic point $y \in$ $V^{*} \otimes \mathbf{C}^{m-n}$ of $\left(\mathfrak{g} \oplus \mathfrak{g l}_{m-n}, \pi^{*} \otimes \Lambda_{1}, V^{*} \otimes \mathbf{C}^{m-n}, B \oplus T_{m-n}\right)$ such that the $\mathfrak{g}$-part of the isotropy subalgebra at $x$ denoted by $\mathfrak{g}_{x}$ coincides with the $\mathfrak{g}$-part of the isotropy subalgebra at $y$ denoted by $\mathfrak{g}_{y}$.

Proof. Suppose that a quadruplet $\left(\mathfrak{g} \oplus \mathfrak{g l}_{n}, \pi \otimes \Lambda_{1}, V \otimes \mathbf{C}^{n}, B \oplus T_{n}\right)$ is prehomogeneous and take arbitrary $n$-vectors $v_{1}, \ldots, v_{n} \in V$ which satisfy $S_{\left(v_{1}, \ldots, v_{n}\right)}=$ $\{(0, \ldots, 0)\}$. Then vectors $v_{1}, \ldots, v_{n} \in V$ are linearly independent. In fact, suppose that there exists scalars $c_{1}, \ldots, c_{n} \in \mathbf{C},\left(c_{1}, \ldots, c_{n}\right) \neq(0, \ldots, 0)$ such that $c_{1} v_{1}+\cdots+c_{n} v_{n}=0$. Then, since $n<m=\operatorname{dim} V$, we have a non-zero element $\phi \in V^{*}$ such that $\left\langle v_{i}, \phi\right\rangle=0$ for $i=1, \ldots, n$ and $\left(c_{1} \phi, \ldots, c_{n} \phi\right)$ is a non-zero element of $S_{\left(v_{1}, \ldots, v_{n}\right)}$. It is a contradiction to the assumption that $S_{\left(v_{1}, \ldots, v_{n}\right)}=$ $\{(0, \ldots, 0)\}$.

Put $U:=\mathbf{C} v_{1}+\cdots+\mathbf{C} v_{n}$ and denote the orthogonal space of $U$ by $U^{\perp}$. Then the space $U^{\perp}$ is a $(m-n)$-dimensional vector subspace of $V^{*}$. Take an arbitrary basis of $U^{\perp}$ and denote it by $\phi_{1}, \ldots, \phi_{m-n} \in V^{*}$. Then $\phi_{1}, \ldots, \phi_{m-n} \in V^{*}$ satisfy $S_{\left(\phi_{1}, \ldots, \phi_{m-n}\right)}^{*}=\{(0, \ldots, 0)\}$. In fact, suppose that $\left(u_{1}, \ldots, u_{m-n}\right) \in S_{\left(\phi_{1}, \ldots, \phi_{m-n}\right)}^{*}$. 
Then for any $i, j(1 \leq i, j \leq m-n)$, we have

$$
\begin{aligned}
& \left\langle u_{i}, \phi_{j}\right\rangle=0, \\
& \Phi_{\pi}\left(u_{1} \otimes \phi_{1}+\cdots+u_{m-n} \otimes \phi_{m-n}\right)=0 .
\end{aligned}
$$

It follows from (2.19) that $u_{1}, \ldots, u_{m-n} \in U$ and thus there exist scalars $c_{k, l} \in \mathbf{C}$ $(1 \leq k \leq m-n, 1 \leq l \leq n)$ which satisfy

$$
u_{k}=c_{k, 1} v_{1}+\cdots+c_{k, n} v_{n} \quad(1 \leq k \leq m-n) .
$$

Then it follows from (2.20) that

$$
\sum_{1 \leq l \leq n} \Phi_{\pi}\left(v_{l} \otimes\left(\sum_{l \leq k \leq m-n} c_{k, l} \phi_{k}\right)\right)=0 .
$$

Thus we have $\quad\left(\left(\sum_{1 \leq k \leq m-n} c_{k, 1} \phi_{k}\right), \ldots,\left(\sum_{1 \leq k \leq m-n} c_{k, n} \phi_{k}\right)\right) \in S_{\left(v_{1}, \ldots, v_{n}\right)}=$ $\{(0, \ldots, 0)\}$. Since $\phi_{1}, \ldots, \phi_{m-n}$ is linearly independent, we have $c_{k, l}=0$ for any $k, l$ and thus $\left(u_{1}, \ldots, u_{n}\right)=(0, \ldots, 0)$. Therefore we have $S_{\left(\phi_{1}, \ldots, \phi_{m-n}\right)}^{*}=$ $\{(0, \ldots, 0)\}$ and thus $\left(\mathfrak{g} \oplus \mathfrak{g l}_{m-n}, \pi^{*} \otimes \Lambda_{1}, V^{*} \otimes \mathbf{C}^{m-n}, B \oplus T_{m-n}\right)$ is also prehomogeneous. Conversely, if $\left(\mathfrak{g} \oplus \mathfrak{g l}_{m-n}, \pi^{*} \otimes \Lambda_{1}, V^{*} \otimes \mathbf{C}^{m-n}, B \oplus T_{m-n}\right)$ is prehomogeneous, then $\left(\mathfrak{g} \oplus \mathfrak{g l}_{m-(m-n)},\left(\pi^{*}\right)^{*} \otimes \Lambda_{1},\left(V^{*}\right)^{*} \otimes \mathbf{C}^{m-(m-n)}, B \oplus T_{m-(m-n)}\right)$ $=\left(\mathfrak{g} \oplus \mathfrak{g l}_{n}, \pi \otimes \Lambda_{1}, V \otimes \mathbf{C}^{n}, B \oplus T_{n}\right)$ is also prehomogeneous.

Next, we put $x:=v_{1} \otimes e_{1}+\cdots+v_{n} \otimes e_{n} \in V \otimes \mathbf{C}^{n}$ and $y:=\phi_{1} \otimes e_{1}+\cdots+$ $\phi_{m-n} \otimes e_{m-n} \in V^{*} \otimes \mathbf{C}^{m-n}$. Let us show that we have $\mathfrak{g}_{x}=\mathfrak{g}_{y}$. A necessary and sufficient condition for an element $a \in \mathfrak{g}$ to belong to $\mathfrak{g}_{x}$ is that there exists an element $A=\sum_{1 \leq i, j \leq n} b_{i j} E_{i j} \in \mathfrak{g l}_{n}$ which satisfies

$$
\begin{aligned}
0 & =\left(\pi \otimes \Lambda_{1}\right)(a, A) \cdot x \\
& =\left(\pi \otimes \Lambda_{1}\right)(a, A) \cdot \sum_{1 \leq i \leq n} v_{i} \otimes e_{i} \\
& =\sum_{1 \leq i \leq n}\left(\pi(a) v_{i}+\sum_{1 \leq j \leq n} b_{i j} v_{j}\right) \otimes e_{i} .
\end{aligned}
$$

Therefore we have

$$
\mathfrak{g}_{x}=\{a \in \mathfrak{g} \mid \pi(a) U \subset U\}
$$

Similarly we have

$$
\mathfrak{g}_{y}=\left\{a \in \mathfrak{g} \mid \pi^{*}(a) U^{\perp} \subset U^{\perp}\right\} .
$$

By an easy calculation, we can obtain $\mathfrak{g}_{x}=\mathfrak{g}_{y}$. This completes the proof. 


\section{References}

[1] N. Bourbaki. Lie groups and Lie algebras. Springer, Berlin, 1989.

[2] H. Rubenthaler. Espaces préhomogènes de type parabolique. Lect. Math. Kyoto Univ. 14 (1982), 189-221.

[3] H. Rubenthaler. Espaces préhomogènes de type parabolique. Thèse d'Etat, Université de Strasbourg, (1982).

[ 4 ] H. Rubenthaler. Algèbres de Lie et espaces préhomogènes, Travaux en cours. Hermann, Paris, 1992.

[5] H. Rubenthaler. Graded Lie algebras associated to a representation of a quadratic algebra. arXiv:1410.0031v1 (2014).

[6] N. Sasano. Lie algebras generated by Lie modules. Kyushu Journal of Mathematics. vol. 68 No. 2 (2014), 377-403.

[ 7 ] M. Sato and T. Kimura. A classification of irreducible prehomogeneous vector spaces and their relative invariants. Nagoya Math. J. 65 (1977), 1-155.

Institute of Mathematics-for-Industry

Kyushu University

744, Motooka, Nishi-ku, Fukuoka 819-0395

Japan

E-mail:n-sasano@math.kyushu-u.ac.jp 